\begin{tabular}{|c|l|}
\hline Title & Entropy production by domain wall decay in the NMSSM \\
\hline Author(s) & Hattori, Hironori; Kobay ashi, Tatsuo; Omoto, Naoya; Seto, O samu \\
\hline Citation & $\begin{array}{l}\text { Physical review D, 92(10), 103518 } \\
\text { https://doi.org/10.1103/PhysRevD.92.103518 }\end{array}$ \\
\hline Issue Date & 2015-11-24 \\
\hline Doc URL & http://hdl.handle.net/2115/60522 \\
\hline Rights & @2016 A merican Physical Society \\
\hline Type & article \\
\hline File Information & PhysRevD.92.103518.pdf \\
\hline
\end{tabular}

Instructions for use 
PHYSICAL REVIEW D 92, 103518 (2015)

\title{
Entropy production by domain wall decay in the NMSSM
}

\author{
Hironori Hattori, Tatsuo Kobayashi, and Naoya Omoto \\ Department of Physics, Hokkaido University, Sapporo 060-0810, Japan \\ Osamu Seto \\ Department of Life Science and Technology, Hokkai-Gakuen University, \\ Sapporo 062-8605, Japan \\ (Received 22 October 2015; published 24 November 2015)
}

\begin{abstract}
We consider domain walls in the $Z_{3}$ symmetric next-to-minimal supersymmetric standard model. The spontaneous $Z_{3}$ discrete symmetry breaking produces domain walls, and the stable domain walls are problematic. Thus, we assume the $Z_{3}$ symmetry is slightly but explicitly broken and the domain walls decay. Such a decay causes a large late-time entropy production. We study its cosmological implications on unwanted relics such as the moduli, gravitino, lightest superparticle, and axion.
\end{abstract}

DOI: 10.1103/PhysRevD.92.103518

PACS numbers: 98.80.Cq, 11.27.+d, 12.60.Jv

\section{INTRODUCTION}

Supersymmetric extension of the standard model (SM) is one of candidates for $\mathrm{TeV}$-scale physics, because supersymmetry (SUSY) can stabilize a large hierarchy. The minimal supersymmetric standard model (MSSM) is quite interesting because of its minimality, and various phenomenological aspects have been studied. However, from a theoretical point of view, it has a problem. The MSSM includes supersymmetric mass terms of Higgs superfields, $H_{u}$ and $H_{d}$, i.e., the so-called $\mu$ term, $\mu H_{u} H_{d}$, in the superpotential. It must be comparable with soft SUSY breaking masses in order to realize successfully the electroweak symmetry breaking. However, the $\mu$ term and soft SUSY breaking terms, in general, have different origins from each other. Why are these comparable with each other? That is the socalled $\mu$-problem [1].

The next-to-minimal supersymmetric standard model (NMSSM) is an extension of the MSSM by adding a singlet superfield $S$ [2] (for a review, see Ref. [3]). Then the NMSSM superpotential has $\lambda S H_{u} H_{d}$. Also, we impose the $Z_{3}$ symmetry, which forbids dimensionful parameters in the superpotential. Dimensionful parameters appear only as soft SUSY breaking parameters. Thus, vacuum expectation values (VEVs) of Higgs and singlet fields are determined by soft SUSY breaking terms. That is, the $\mu$-problem is solved, and the effective $\mu$ term is generated as $\mu=\lambda\langle S\rangle$.

In the NMSSM, the Higgs sector as well as the neutralino sector has a richer structure than one in the MSSM, because of the inclusion of the singlet superfield $S$. Also, the NMSSM can raise the SM-like Higgs boson mass. At any rate, heavier superpartner masses such as $\mathcal{O}(1)-$ $\mathcal{O}(10) \mathrm{TeV}$ may be favorable. We may need fine-tuning to realize a little hierarchy between the electroweak scale and SUSY breaking scale. However, such a fine-tuning can be improved in a certain mediation mechanism, e.g., in the TeV-scale mirage mediation scenario [4]. ${ }^{1}$

The $Z_{3}$ symmetry is important to forbid dimensionful parameters in the superpotential and to solve the $\mu$-problem. However, it is problematic. VEVs of the Higgs scalar and singlet break spontaneously the $Z_{3}$ symmetry. In general, when a discrete symmetry is spontaneously broken, domain walls appear. They would dominate the energy density of the Universe and change the standard cosmology drastically. Thus, the exact $Z_{3}$ symmetry and the stable domain walls are problematic [9]. See for the NMSSM [10].

Here, we assume that the $Z_{3}$ symmetry is broken explicitly, but its breaking size is much smaller than the electroweak scale. Then the domain walls are unstable. They may dominate the energy density of the Universe at a certain period but decay. It has important effects on thermal history. (See, e.g., Ref [11].) In this paper, we study implications of unstable domain walls in the NMSSM. In general, SUSY models have other problems due to the moduli, gravitino, and lightest superparticle (LSP). For example, in the gravity mediation scenario, moduli and gravitino masses would be comparable with masses of superpartners in the visible sector. When those are of $\mathcal{O}(1)-\mathcal{O}(10) \mathrm{TeV}$, they affect successful big bang nucleosynthesis (BBN), that is, the socalled moduli problem and gravitino problem. They could be diluted by decay of domain walls [12]. Furthermore, even if the moduli and gravitino are heavier than superpartners in the visible sector, that would lead to another problem. Indeed, in the mirage mediation mechanism [6], the gravitino is heavier by $\mathcal{O}\left(8 \pi^{2}\right)$ than superpartners in the visible sector, and the modulus is also heavier by $\mathcal{O}\left(8 \pi^{2}\right)$ than the gravitino. In such a case, the moduli decay into the gravitino

\footnotetext{
${ }^{1}$ See for phenomenological aspects of MSSM in the TeV-scale mirage mediation scenario [5] and for generic mirage mediation [6-8].
} 
with a large rate and the gravitino decays into the LSP. This overproduces nonthermally the LSP [13]. We need to dilute the moduli, gravitino, and LSP. Also, in some other scenarios, the LSP such as binolike neutralino has a large thermal relic density. The decay of domain walls, which was mentioned above, can produce a large entropy and dilute moduli and dark matter candidates in the NMSSM.

This paper is organized as follows. In Sec. II, we study the domain wall solution in the NMSSM. In Sec. III, we study cosmological evolution of unstable domain walls. In Secs. IV-V, we study implications of the domain wall decay in two scenarios. Section VI is devoted to conclusion and discussion.

\section{DOMAIN WALL SOLUTION IN THE NMSSM}

\section{A. Domain wall solution in the $Z_{3}$ symmetric NMSSM}

We briefly review a domain wall solution of the Higgs potential in the $Z_{3}$ symmetric NMSSM. ${ }^{2}$ We adopt the convention for $H_{u}, H_{d}$ and $S$ that the superfield and its lowest component are written by the same letter. The superpotential terms including only $H_{u}, H_{d}$ and $S$ are written as

$$
W_{\mathrm{Higgs}}=\lambda S H_{u} H_{d}+\frac{\kappa}{3} S^{3},
$$

where the $Z_{3}$ symmetry is imposed as mentioned. The scalar potential is written by

$$
V_{\mathrm{Higgs}}=\sum_{\phi_{i}=H_{u}, H_{d}, S}\left|\frac{\partial W}{\partial \phi_{i}}\right|^{2}+V_{D}+V_{\mathrm{soft}},
$$

where $V_{D}$ is the D-term potential due to $S U(2) \times U(1)_{Y}$ and $V_{\text {soft }}$ denotes the soft SUSY breaking terms,

$$
\begin{aligned}
V_{\text {soft }}= & m_{H_{u}}^{2}\left|H_{u}\right|^{2}+m_{H_{d}}^{2}\left|H_{u}\right|^{2}+\frac{1}{3} \kappa A_{\kappa} S^{3}+\lambda A_{\lambda} H_{u} H_{d} S \\
& + \text { H.c. }
\end{aligned}
$$

Only the neutral components develop their VEVs, and their scalar potential is written explicitly by

$$
\begin{aligned}
V_{\mathrm{Higgs}}= & \left|\kappa S^{2}-\lambda H_{u}^{0} H_{d}^{0}\right|^{2}+m_{H_{u}}^{2}\left|H_{u}^{0}\right|^{2}+m_{H_{d}}^{2}\left|H_{d}^{0}\right|^{2} \\
& +m_{S}^{2}|S|^{2}+|\lambda|^{2}|S|^{2}\left(\left|H_{d}^{0}\right|^{2}+\left|H_{u}^{0}\right|^{2}\right) \\
& +\frac{g^{2}+g^{2}}{8}\left(\left|H_{u}^{0}\right|^{2}-\left|H_{d}^{0}\right|^{2}\right)^{2}+\frac{1}{3} \kappa A_{\kappa} S^{3} \\
& -\lambda A_{\lambda} H_{u}^{0} H_{d}^{0} S+\text { H.c. },
\end{aligned}
$$

\footnotetext{
${ }^{2}$ The full scalar potential includes superpartners of quarks and leptons, and it has several unrealistic vacua. We assume that taken SUSY breaking parameters in the full potential satisfy the condition to avoid such unrealistic vacua. (See, e.g., Ref. [14] and references therein.)
}

where $g$ and $g^{\prime}$ are the $S U(2)$ and $U(1)_{Y}$ gauge couplings, respectively. Here, we assume that all of $\lambda, \kappa, A_{\lambda}$, and $A_{\kappa}$ are real.

The potential minima are obtained by analyzing the stationary conditions,

$$
\frac{\partial V_{\mathrm{Higgs}}}{\partial H_{u}^{0}}=\frac{\partial V_{\mathrm{Higgs}}}{\partial H_{d}^{0}}=\frac{\partial V_{\mathrm{Higgs}}}{\partial S}=0
$$

and these VEVs lead to the successful electroweak symmetry breaking, where the effective $\mu$ term is obtained as $\mu=\lambda\langle S\rangle$. Since the scalar potential has the $Z_{3}$ symmetry, three vacua are degenerate,

$$
\left(\langle S\rangle,\left\langle H_{u}^{0}\right\rangle,\left\langle H_{d}^{0}\right\rangle\right)=\left(v_{s} e^{2 \pi i m / 3}, v_{u} e^{2 \pi i m / 3}, v_{d} e^{2 \pi i m / 3}\right),
$$

with $m=0,1,2$, where all $v_{s}, v_{u}$, and $v_{d}$ are real with $v=\sqrt{v_{u}^{2}+v_{d}^{2}} \simeq 174 \mathrm{GeV}$. One of three degenerate vacua is selected in the vacuum, and then the $Z_{3}$ symmetry is broken spontaneously. Then the domain walls are generated.

First, we study the domain wall solution [15]. We fix field values of radial directions of $S, H_{u}$, and $H_{d}$, and discuss a field equation for the phase degree of freedom $\phi$,

$$
\left(S, H_{u}^{0}, H_{d}^{0}\right)=\left(v_{s} e^{\mathrm{i} \phi}, v_{u} e^{\mathrm{i} \phi}, v_{d} e^{\mathrm{i} \phi}\right) .
$$

The potential of $\phi$ can be obtained from $V_{\text {Higgs }}$ as

$V(\phi)=-2\left(\frac{1}{3}\left|\kappa A_{\kappa} v_{s}^{3}\right|+\lambda A_{\lambda} v_{s} v_{u} v_{d}\right) \cos (3 \phi)+V_{0}$,

where $V_{0}$ denotes $\phi$-independent terms. The first term would be dominant when $A_{\kappa} \sim A_{\lambda}, \lambda \sim \kappa$, and $v_{s}^{2} \gg v_{u} v_{d}$. Also, the kinetic term of $\phi$ is written by

$$
\mathcal{L}_{\text {kinetic }}(\phi)=\eta^{2}\left(\partial_{\mu} \phi\right)\left(\partial^{\mu} \phi\right),
$$

with $\eta^{2}=v_{s}^{2}+v_{u}^{2}+v_{d}^{2}$.

For simplicity, we consider a planar domain wall orthogonal to the $z$ axis, $\phi(z)$. Then the field equation,

$$
\partial_{\mu} \frac{\partial \mathcal{L}_{\text {kinetic }}}{\partial_{\mu}(\partial \phi)}+\frac{\partial V_{\mathrm{VEV}}}{\partial \phi}=0,
$$

can be written by

$$
\frac{\mathrm{d}^{2} \phi}{\mathrm{d} z^{2}}-\frac{1}{3 B^{2}} \sin (3 \phi)=0,
$$

with

$$
\left(\frac{1}{B}\right)^{2}=\frac{9\left(\left|\frac{1}{3} \kappa A_{\kappa} v_{s}^{3}\right|+\lambda A_{\lambda} v_{s} v_{u} v_{d}\right)}{\eta^{2}} .
$$




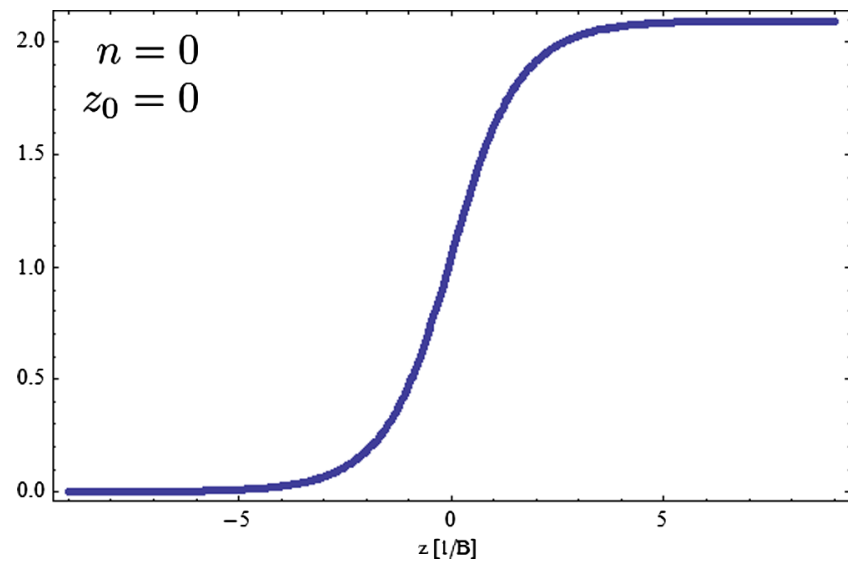

FIG. 1 (color online). The phase of scaler field $\left(S(z), H_{u}(z)\right.$, $\left.H_{d}(z)\right)$ of the planer domain wall solution. Here we take $n=0$, $z_{0}=0$, and normalize the $\mathrm{z}$ axis by $1 / B$ [Eq. (12)].

The first term in the numerator of the left-hand side of Eq. (12) is dominant when $v_{s}^{2} \gg v_{u} v_{d}$. We set the boundary condition such that $\phi=2 \pi n / 3$ at $z \rightarrow-\infty$ and $\phi=$ $2 \pi(n+1) / 3$ at $z \rightarrow+\infty$ with $n=0,1,2$. By solving the above field equation with this boundary condition, the domain wall solution is derived as

$$
\phi=\frac{2 n \pi}{3}+\frac{4}{3} \arctan \left(\mathrm{e}^{ \pm \frac{1}{B}\left(z-z_{0}\right)}\right),
$$

where $B$ corresponds to the width of the domain wall. Figure 1 shows this solution for $n=0$.

Now we can estimate the domain wall tension,

$$
\begin{aligned}
\sigma & =\int d z \rho_{\text {wall }}(z) \\
& =\int d z\left(\left|\frac{\mathrm{d} S}{\mathrm{~d} z}\right|^{2}+\left|\frac{\mathrm{d} H_{u}^{0}}{\mathrm{~d} z}\right|^{2}+\left|\frac{\mathrm{d} H_{d}^{0}}{\mathrm{~d} z}\right|^{2}+V(\phi)\right) \\
& =\frac{16}{9} \frac{\eta^{2}}{B} .
\end{aligned}
$$

Thus, we can estimate

$$
\sigma \simeq \frac{16}{3 \sqrt{3}} v_{s}^{2} \sqrt{\kappa A_{\kappa} v_{s}}=\frac{16}{3 \sqrt{3}} \frac{\mu^{2}}{\lambda^{2}} \sqrt{\frac{\kappa}{\lambda} A_{\kappa} \mu},
$$

for $v_{s}^{2} \gg v_{u} v_{d}$. The size of $\mu$ is of the SUSY breaking scale. ${ }^{3}$ The couplings $\lambda$ and $\kappa$ must be of $\mathcal{O}(0.1)$ or less at the electroweak scale such that they do not blow up below a high

\footnotetext{
${ }^{3}$ When $\mu$ is much larger than the electroweak scale, we have the fine-tuning problem to derive the Z-boson mass $m_{Z}$ from $m_{H_{u}}^{2}$, $\mu$, and $m_{H_{d}}^{2}$. However, in a certain mediation such as the TeVscale mirage mediation contributions due to $\mu$ and $m_{H_{d}^{2}}$ cancel each other in $m_{Z}$, and $m_{Z}$ is independent of $\mu$. Without severe fine-tuning $\mu$ can be larger than the electroweak scale, e.g., $\mu=$ $\mathcal{O}(1) \mathrm{TeV}[4]$.
}

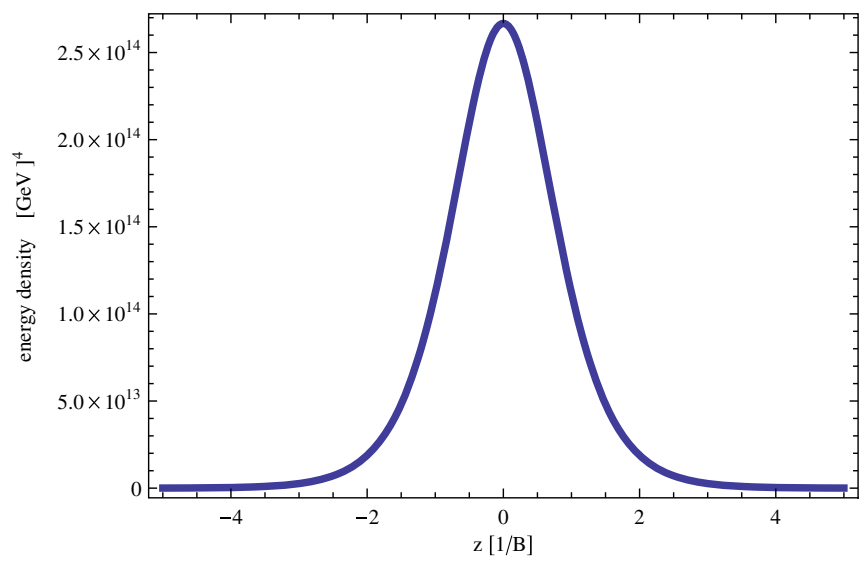

FIG. 2 (color online). Spatial configuration of a domain wall energy density for $\lambda=\kappa=0.01, \quad A_{\lambda}=A_{\kappa}=10 \mathrm{TeV}$, $\mu=1 \mathrm{TeV}, \tan \beta=10$. The $\mathrm{z}$ axis is normalized by $1 / B$.

energy scale such as the grand unified theory (GUT) or Planck scale. Thus, the size of $\sigma^{1 / 3}$ would be of the SUSY breaking scale or larger. Figure 2 shows an example of $\rho_{\mathrm{DW}}(z)$.

\section{B. Decaying domain wall by $\boldsymbol{Z}_{\mathbf{3}}$ breaking}

Formed domain walls are stretched by the cosmic expansion and smoothed by interactions with particles in the background thermal plasma. The energy density of domain walls $\rho_{\text {DW }}$ and its pressure $p_{\text {DW }}$ can be read from the averaged energy momentum tensor of domain walls. The equation of state of domain walls is given by

$$
p_{\mathrm{DW}}=\left(v^{2}-\frac{2}{3}\right) \rho_{\mathrm{DW}},
$$

with $v$ being the averaged velocity of walls [16]. The dynamics depends on $v$. In one extremal limit, nonrelativistic limit, or static limit with $v=0$, the energy density behaves as

$$
\rho_{\mathrm{DW}} \propto a^{-1},
$$

where $a(t)$ is the scale factor of the Universe. Such a domain wall network is sometime referred to as a "frustrated domain wall." Such a frustrated domain wall dominated Universe causes accelerated expansion because of $w=p / \rho=-2 / 3<-1 / 3$. On the other hand, for $v^{2} \geq$ $1 / 3$ where $w \geq-1 / 3$ is realized, the cosmic expansion is not accelerating.

In fact, the dynamics of domain walls has been investigated and many detailed numerical simulations show that the dynamics of the domain wall network is relaxed at a late time to the so-called scaling regime, where the typical length scale $\xi$ of the system stays of the Hubble radius $H^{-1}$ [17-22]. Then, the energy density of domain walls also scales as [22] 


$$
\rho_{\mathrm{DW}} \simeq \frac{\sigma}{t}
$$

The energy density of the domain wall decreases slower than any other "matter" or radiation in the scaling solution. Thus, at some point, the energy density of domain walls dominates that in the Universe. This is the domain wall problem [9].

Thus, the stable domain wall in the $Z_{3}$ symmetric NMSSM is problematic [10]. In this paper, we consider a tiny but explicit breaking of the $Z_{3}$ discrete symmetry so that domain walls might have a long lifetime but finally decay. In fact, the decay of domain walls after domain wall domination has an interesting cosmological implication, namely the dilution of unwanted relics by late-time entropy production [12].

Few numerical detailed studies on dynamics of the domain wall network in a domain wall dominated Universe have been done. Hence, the domain wall dynamics in a domain wall dominated Universe after its scaling behavior is uncertain. One likely possibility is that the scale of the system remains of the order of the Hubble radius as in the scaling regime after domain wall domination. This can be realized for the equation of state $w \simeq-1 / 3$. Thus, in most of the following analysis, we assume this. On the other hand, there is another possibility that the dynamics after the domination would be frozen as suggested in Ref. [20], where $\xi \propto a(t)$ and $\rho \propto a(t)^{-1}$ are realized as in the nonrelativistic limit. We briefly discuss results for this latter case, too.

Before closing this subsection, we briefly note some examples of the $Z_{3}$ symmetry breaking in the literature for information. In Ref. [23], Panagiotakopoulos and Tamvakis proposed adding extra symmetries, which consistently allows one to induce a tiny enough tadpole term

$$
\Delta V \sim \frac{1}{\left(16 \pi^{2}\right)^{n}} m_{\mathrm{SUSY}}^{3}\left(S+S^{*}\right),
$$

where $m_{\text {SUSY }}$ is a soft SUSY breaking mass and $n$ is a power of loop inducing this term in the scalar potential and the degeneracy of vacua is resolved. Hamaguchi et al. proposed another solution by introducing hidden QCD theory, where the $Z_{3}$ symmetry becomes anomalous and is broken by quantum effects [24]. In such a minor extension of $Z_{3}$ symmetric NMSSM, the domain walls become unstable. Since the size of the $Z_{3}$ breaking term is highly model dependent and the main purpose of this paper is to study cosmological effects of late-time domain wall decay, the decay rate of a domain wall $\Gamma_{\mathrm{DW}}$, which also parametrizes the size of the $Z_{3}$ symmetry breaking, is treated as a free parameter. Throughout this paper, in order to connect successful BBN, we take the domain wall decay temperature

\footnotetext{
${ }^{4}$ In the static limit $v=0$, it is even slower.
}

$T_{d}$ of a few $\mathrm{MeV}$. We note that the lower bound of the reheating temperature by late decay objects is about a few MeV [25-27].

\section{COSMOLOGICAL EVOLUTION OF UNSTABLE DOMAIN WALLS}

When doublet and/or singlet Higgs fields develop the VEVs, domain walls are formed. As mentioned above, after certain dynamics, the domain wall network would be relaxed to be in the scaling solution. In the scaling regime, the energy density of domain walls is given by

$$
\rho_{\mathrm{DW}} \simeq \sigma H \text {. }
$$

\section{A. Matter-dominated era to domain wall dominated era}

The first case we consider is that, at the domain wall formation time $H_{i}^{-1}$, the Universe is dominated by the energy density of a matter $\rho_{M}$ such as a long-lived coherent oscillating moduli field. In the scaling solution of domain wall, the energy density of domain walls relative to that of the background increases and eventually dominates the Universe. The domain wall energy density becomes equal to that of the matter at $H_{\mathrm{eq}}^{-1}$, which is estimated with Eq. (19) as

$$
H_{\mathrm{eq}} \simeq \frac{\sigma}{3 M_{P}^{2}}
$$

where $M_{P}$ is the reduced Planck mass. The condition that domain walls indeed dominate the Universe before they decay is expressed as

$$
H_{\text {eq }}>\Gamma_{\text {DW }}
$$

After $H_{\text {eq }}$, the domain walls dominate the energy density.

At the domain wall decay time $\Gamma_{\mathrm{DW}}^{-1}$, the ratio of these energy densities is estimated as

$$
\left.\frac{\rho_{M}}{\rho_{\mathrm{DW}}}\right|_{\Gamma_{\mathrm{DW}}}=\left(\frac{\Gamma_{\mathrm{DW}}}{H_{\mathrm{eq}}}\right)
$$

from $a \propto t$, where we assume $\rho_{\text {DW }} \propto a^{-2}$ during the domain wall domination between $H_{\mathrm{eq}}$ and $\Gamma_{\mathrm{DW}}$. After the domain walls decay, the energy density of the matter is diluted as

$$
\frac{\rho_{M}}{s}=\frac{3 T_{d}}{4}\left(\frac{\Gamma_{\mathrm{DW}}}{H_{\mathrm{eq}}}\right) \simeq \frac{3 T_{d}}{4}\left(\frac{\pi^{2} g_{*}\left(T_{d}\right) T_{d}^{4}}{10} \frac{M_{P}^{2}}{\sigma^{2}}\right)^{1 / 2}
$$

for the case that the domain wall decays earlier than the matter does. Here, $g_{*}$ is the number of relativistic degrees of freedom. 


\section{B. Radiation-dominated era to domain wall dominated era}

Next, we discuss the case that domain walls are formed in a radiation-dominated universe. Both energy densities become comparable with each other at

$$
H_{\mathrm{eq}} \simeq \frac{\sigma}{3 M_{P}^{2}},
$$

since domain walls are in the scaling solution. The entropy density ratio of after to before domain wall decay is given by

$$
\begin{aligned}
\Delta & =\frac{s_{\text {after }}}{s_{\text {before }}} \simeq \frac{T_{\mathrm{eq}}}{T_{d}}\left(\frac{H_{\mathrm{eq}}}{\Gamma_{\mathrm{DW}}}\right) \\
& \simeq\left(\frac{10 \sigma^{2}}{\pi^{2} g_{*}\left(T_{d}\right) T_{d}^{4} M_{P}^{2}}\right)^{3 / 4}\left(\frac{g_{*}\left(T_{d}\right)}{g_{*}\left(T_{\mathrm{eq}}\right)}\right)^{1 / 4},
\end{aligned}
$$

for $\Delta \gg 1$. We can obtain an entropy production

$$
\Delta \simeq 10\left(\frac{\sigma^{1 / 3}}{50 \mathrm{TeV}}\right)^{9 / 2}\left(\frac{2 \mathrm{MeV}}{T_{d}}\right)^{3}
$$

One might think that the tension of about $100 \mathrm{TeV}$ looks somewhat too large. However, for instance, in the MSSMlike region of the NMSSM with $\lambda \sim \kappa \ll 1$ and $v_{s} \gg v$, the domain wall tension

$$
\sigma \simeq \frac{16}{3} \sqrt{\frac{2}{3}} \kappa v_{s}^{3}
$$

can be of such an order with $\lambda \sim \kappa \sim 10^{-2}$ and $v_{s} \sim 100 \mathrm{TeV}$. Those result in the effective $\mu$ term and the singlino mass of about $1 \mathrm{TeV}$. Figure 3 shows the entropy density ratio of after to before domain wall decay for $\lambda=\kappa=0.01, T_{d}=3 \mathrm{MeV}$. The ratio increases as $\mu$ and $A_{\kappa}$ increase.

Such a large late-time entropy production can dilute unwanted relics such as the gravitino, overproduced LSP, as well as axion.

\section{Nonrelativistc domain wall during the domination}

Here, we note resultant quantities if the domain wall energy density scales as $a^{-1}$ during the domination.

\section{Matter-dominated era to domain wall dominated era}

At the domain wall decay time $\Gamma_{\mathrm{DW}}^{-1}$, the ratio of these energy densities is estimated as

$$
\left.\frac{\rho_{M}}{\rho_{\mathrm{DW}}}\right|_{\Gamma_{\mathrm{DW}}}=\left(\frac{\Gamma_{\mathrm{DW}}}{H_{\mathrm{eq}}}\right)^{4},
$$

from $H \propto a^{-1 / 2}$, where we assume $\rho_{\mathrm{DW}} \propto a^{-1}$ during the domain wall domination between $H_{\mathrm{eq}}$ and $\Gamma_{\mathrm{DW}}$. After the domain walls decay, the energy density of the matter is diluted as

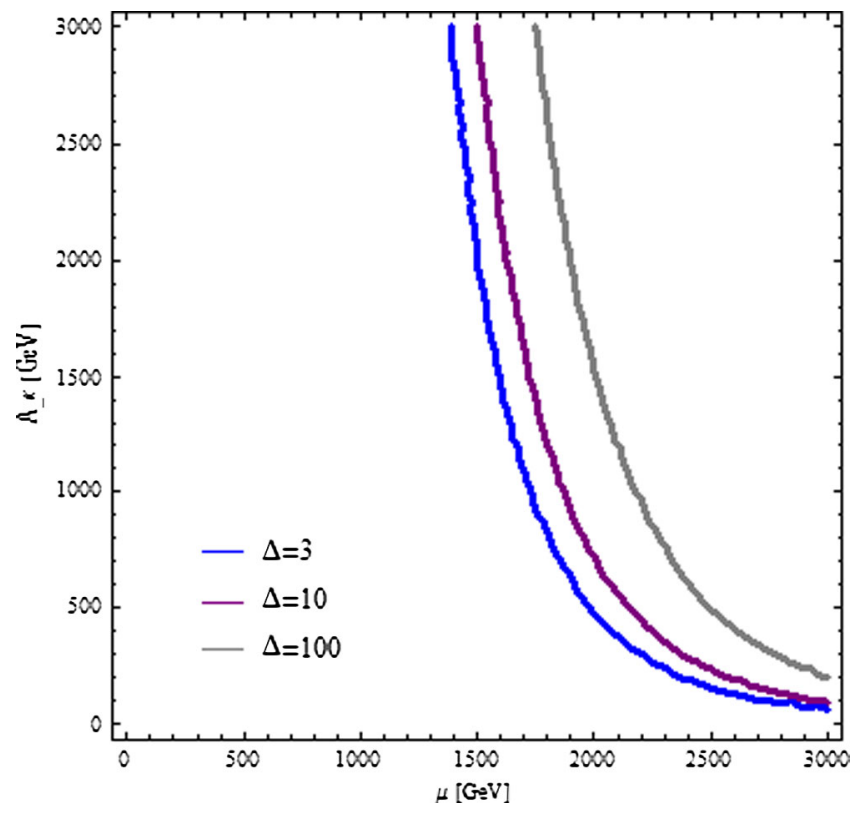

FIG. 3 (color online). The entropy density ratio $\Delta$ of after to before domain wall decay in the radiation-dominated era to domain wall dominated era for $\lambda=\kappa=0.01, T_{d}=3 \mathrm{MeV}$.

$$
\frac{\rho_{M}}{s}=\frac{3 T_{d}}{4}\left(\frac{\Gamma_{\mathrm{DW}}}{H_{\mathrm{eq}}}\right)^{4} \simeq \frac{3 T_{d}}{4}\left(\frac{\pi^{2} g_{*}\left(T_{d}\right) T_{d}^{4}}{10} \frac{M_{P}^{2}}{\sigma^{2}}\right)^{2}
$$

for the case that the domain wall decays earlier than the matter does.

\section{Radiation-dominated era to domain wall dominated era}

Assuming $\rho_{\text {DW }} \propto a^{-1}$ during the domain wall domination, the entropy density ratio of after to before domain wall decay is given by

$$
\begin{aligned}
\Delta & =\frac{s_{\text {after }}}{S_{\text {before }}} \simeq \frac{T_{\text {eq }}}{T_{d}}\left(\frac{H_{\mathrm{eq}}}{\Gamma_{\mathrm{DW}}}\right)^{4} \\
& \simeq\left(\frac{10 \sigma^{2}}{\pi^{2} g_{*}\left(T_{d}\right) T_{d}^{4} M_{P}^{2}}\right)^{9 / 4}\left(\frac{g_{*}\left(T_{d}\right)}{g_{*}\left(T_{\mathrm{eq}}\right)}\right)^{1 / 4},
\end{aligned}
$$

for $\Delta \gg 1$. We can obtain an entropy production

$$
\Delta \simeq 600\left(\frac{\sigma^{1 / 3}}{50 \mathrm{TeV}}\right)^{27 / 2}\left(\frac{2 \mathrm{MeV}}{T_{d}}\right)^{9}
$$

\section{COSMOLOGICAL IMPLICATIONS}

In this section, we study implications of the NMSSM domain wall decay to some relics in several models. 


\section{A. Thermal relic WIMP LSP such as singlino or sneutrino}

Weakly interacting massive particles (WIMPs) have been regarded as a promising dark matter candidate in our Universe. In the NMSSM, the neutralino is the candidate [3]. In a right-hand neutrino extended model, the right-hand sneutrino also becomes a WIMP dark matter candidate [28]. Since the WIMP thermal relic abundance is inversely proportional to its thermal averaged annihilation cross section $\langle\sigma v\rangle$ as

$$
\Omega_{\mathrm{WIMP}} h^{2} \simeq \frac{0.1 \mathrm{pb}}{\langle\sigma v\rangle}
$$

a too-small annihilation cross section leads to overabundant WIMPs. The singlino- or binoike neutralino, or the righthand sneutrino with small couplings, is indeed such a case. The domain wall decay produces extra entropy with the dilution factor (25) and could regulate the WIMP relic abundance to be

$$
\Omega_{\mathrm{WIMP}} h^{2} \frac{1}{\Delta} \simeq 0.1,
$$

even for a small annihilation cross section $\langle\sigma v\rangle \ll 1 \mathrm{pb}$.

\section{B. The moduli problem in the mirage mediation scenario}

Mirage mediation models appear free from the cosmological moduli problem because a moduli mass is quite large. However, the nonthermally produced LSP through a decay chain by way of the gravitino is in fact overabundant. Let us examine whether the domain wall decay dilutes those LSPs.

Moduli decay before the energy density of domain walls dominates the Universe because the moduli decay rate

$$
\Gamma_{\text {moduli }} \simeq \frac{m_{\text {moduli }}^{3}}{8 \pi M_{P}^{2}}
$$

is larger than $H_{\mathrm{eq}}$ given by Eq. (20) in the mirage mediation scenario. At $H \simeq \Gamma_{\text {moduli }}$, the moduli decay at a moduli dominated universe produces gravitinos as

$$
Y_{3 / 2}=\frac{n_{3 / 2}}{s}=B_{3 / 2} \frac{3 T_{D}}{2 m_{\text {moduli }}},
$$

with the branching ratio of moduli decay into gravitinos $B_{3 / 2}=\mathcal{O}(0.01)-\mathcal{O}(1)$ [13], and the Universe becomes radiation dominated. Here $T_{D}$ is the decay temperature of the moduli field given by

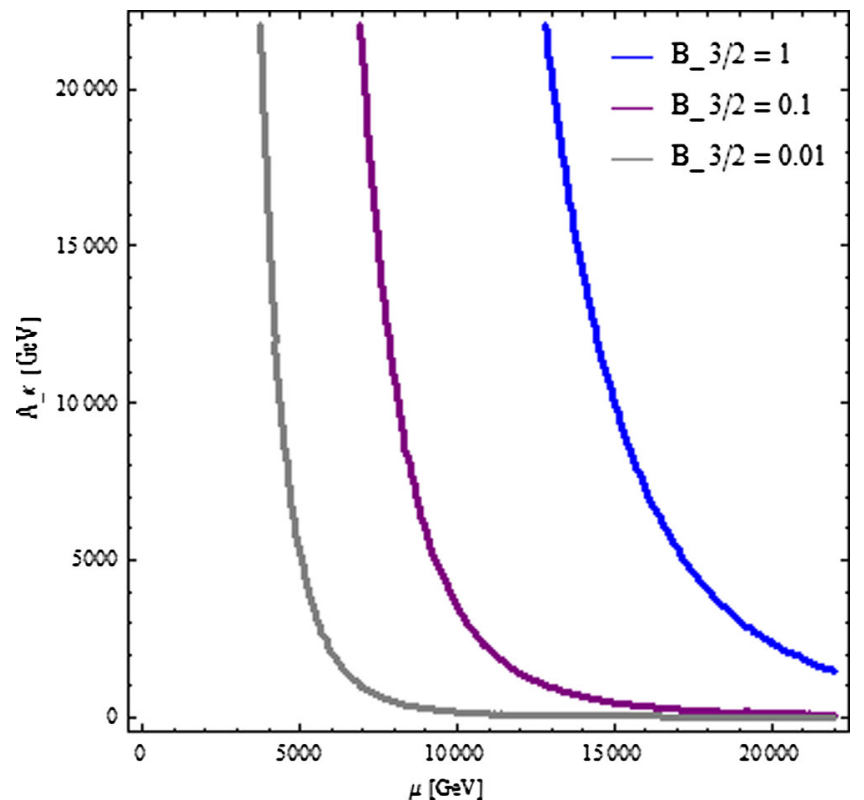

FIG. 4 (color online). The required branching ratio contour to keep $\Omega_{\mathrm{LSP}} h^{2}=0.1$ in the mirage mediation scenario for $\lambda=\kappa=0.01, \quad T_{d}=3 \mathrm{MeV}, \quad m_{\mathrm{LSP}}=100 \mathrm{GeV}, \quad m_{\text {moduli }}=$ $1000 \mathrm{TeV}$. Above each curve, the relic abundance is smaller than $\Omega_{\mathrm{LSP}} h^{2}=0.1$.

$$
3 M_{P}^{2} \Gamma_{\text {moduli }}^{2}=\frac{\pi^{2} g_{*}\left(T_{D}\right)}{30} T_{D}^{4}
$$

The entropy density ratio of after to before domain wall decay is given by Eq. (25). Unstable gravitinos decay into the LSP with $n_{3 / 2}=n_{\mathrm{LSP}}$ due to R-parity conservation. Usually, this leads to the overproduction of the LSP whose abundance exceeds the dark matter abundance. After extra entropy production by the domain wall decay, the resultant final LSP abundance becomes

$$
\frac{\rho_{\mathrm{LSP}}}{s} \simeq \frac{3 m_{\mathrm{LSP}} T_{D}}{2 m_{\text {moduli }}} \frac{B_{3 / 2}}{\Delta} ;
$$

in other words,

$$
\Omega_{\mathrm{LSP}} h^{2} \simeq 4.2 \times 10^{8} \frac{m_{\mathrm{LSP}} T_{D}}{m_{\text {moduli }}} \frac{B_{3 / 2}}{\Delta} \mathrm{GeV}^{-1} .
$$

In Fig. 4, we consider the case that the LSP is the dark matter, and plot $\Omega_{\mathrm{LSP}} h^{2}=0.1$ by using (38). The input parameters are $\lambda=\kappa=0.01, \quad T_{d}=3 \mathrm{MeV}, m_{\mathrm{LSP}}=$ $100 \mathrm{GeV}, m_{\text {moduli }}=1000 \mathrm{TeV}$.

\section{The decay constant of the QCD axion}

Finally, we comment on the QCD axion, $a$, with the decay constant $f_{a}$. After the QCD transition, axions are produced by coherent oscillation, the so-called misalignment mechanism, and it is a good candidate for dark matter 
because its lifetime is much longer than the age of the Universe. Its abundance is proportional to $f_{a}^{7 / 6}$ [29]. The condition $\Omega_{a} \lesssim \Omega_{\mathrm{DM}}$ is rewritten as

$$
f_{a} \lesssim 10^{12} \mathrm{GeV}
$$

$f_{a}$, which is larger than (39), corresponds to the overproduction of axions. Again, the domain wall decay can dilute the axion abundance for such a larger $f_{a}$ [12].

For example, with the dilution (25) by the domain wall decay, the bound on $f_{a}$ is relaxed as

$$
f_{a} \lesssim 10^{16} \mathrm{GeV}
$$

for $\sigma^{1 / 3}=300 \mathrm{TeV}$ and $T_{d}=2 \mathrm{MeV}$.

The GUT scale axion decay constant is allowed, which is remarkable. In superstring theory, the natural decay constant of axionic parts in a closed string moduli would be of the order of the GUT scale or string scale [30]. ${ }^{5}$ Such stringy axions with a larger decay constant can be the QCD axion.

\section{COSMOLOGICAL IMPLICATIONS FOR $w=-1 / 3$ DOMAIN WALLS}

In this section, we study implications of the NMSSM domain wall decay with $w=-1 / 3$ for the moduli problem within the gravity mediation scenario.

Now let us study the dilution of moduli to avoid the moduli problem. After inflation, the moduli would start to oscillate and dominate the energy density of the Universe. They may decay during or after the BBN and change the success of BBN. To avoid such a situation, the energy density of moduli must satisfy

$$
\frac{\rho_{\text {moduli }}}{s} \lesssim c \cdot 3.6 \times 10^{-9} \mathrm{GeV}
$$

where $c \sim 10^{-2}-10^{-4}$ for $10 \mathrm{TeV}$ moduli mass depending on the coupling between the moduli and the gauge field [32]. We use $c=10^{-3}$ in the following analysis.

The decay of domain walls can dilute the moduli density, which is given as

$$
\frac{\rho_{\text {moduli }}}{s} \simeq \frac{3 T_{d}}{4}\left(\frac{\pi^{2} g_{*}\left(T_{d}\right) T_{d}^{4}}{10} \frac{M_{P}^{2}}{\sigma^{2}}\right)^{2}
$$

as derived in Eq. (29). It depends on only $T_{d}$ and tension $\sigma$, which depends on $\lambda, \kappa, A_{\kappa}$, and $\mu$. Imposing the constraint (41) on the resultant abundance (42), we find

\footnotetext{
${ }^{5}$ Even larger decay constants can be obtained in a certain situation (see, e.g., Ref. [31]).
}

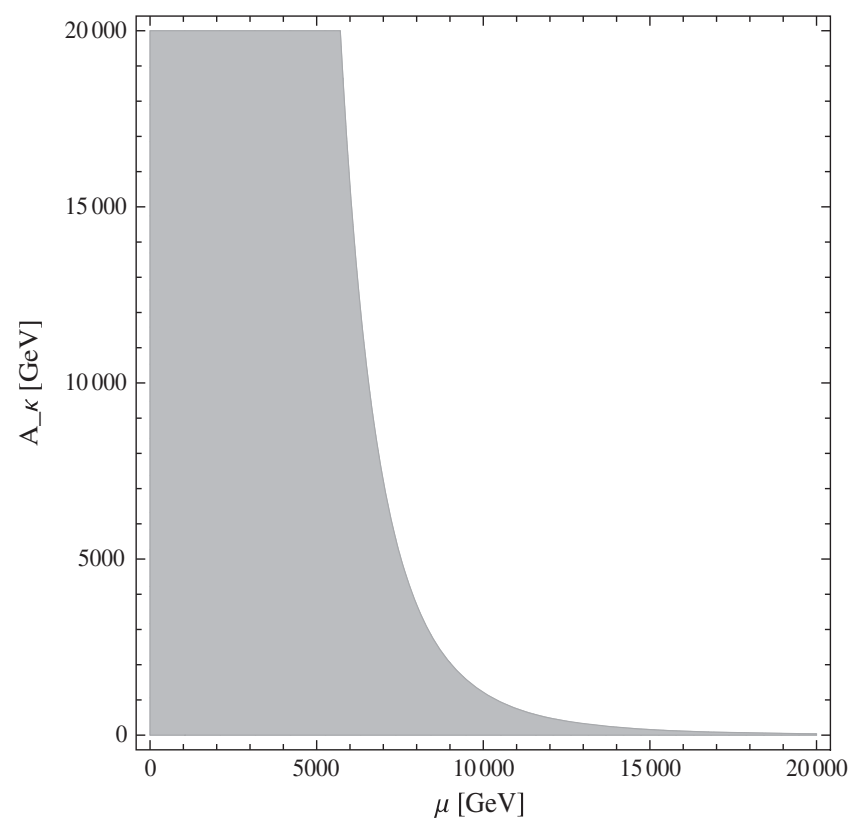

FIG. 5. The bound of the moduli abundance in gravity mediation scenario for $\lambda=\kappa=0.01, T_{d}=3 \mathrm{MeV}$. The gray region is forbidden in the $\left(\mu, A_{\kappa}\right)$ plane.

$$
\sigma^{1 / 3} \gtrsim 220 \mathrm{TeV}\left(\frac{10^{-3}}{c}\right)^{1 / 12}\left(\frac{T_{d}}{3 \mathrm{MeV}}\right)^{3 / 4},
$$

where $g\left(T_{d}\right)=10$ is used.

Figure 5 shows the constraints (41) with (42) for $\lambda=\kappa=0.01, T_{d}=3 \mathrm{MeV}$. The shaded region is excluded by the constraint.

\section{CONCLUSION AND DISCUSSION}

We have studied the cosmological implication of unstable domain walls in the NMSSM. The spontaneous breaking of the $Z_{3}$ discrete symmetry in the NMSSM causes the cosmological domain wall problem. We consider that the $Z_{3}$ symmetry is slightly but explicitly broken and the domain walls decay with the decay temperature $T_{d}$. The domain walls easily dominate the density of the Universe and its decay causes a late-time entropy production, depending on its tension $\sigma$ and $T_{d}$. Such entropy production has significant implications in thermal history. They can dilute unwanted relics such as the moduli, gravitino, LSP, and axion.

We have shown that $T_{d}$ of several $\mathrm{MeV}$ dilute various relics in several scenarios. Those include the thermal WIMP LSP in the gravity mediation model, the nonthermally produced LSP in mirage mediation, and the misalignment produced cold axion in Peccei-Quinn extended models. If the energy density of the domain wall network decreases as $\rho_{\text {Dw }} \propto a^{-1}$ during domain wall domination, the cosmological moduli problem in gravity mediation also might be relaxed. 


\section{ACKNOWLEDGMENTS}

This work was supported in part by the Grant-in-Aid for Scientific Research Grants No. 25400252 and No. 26247042 (T. K.) and the Innovative Areas Grant No. 26105514 (O. S.) from the Ministry of Education, Culture, Sports, Science and Technology in Japan.

[1] J. E. Kim and H. P. Nilles, The mu problem and the strong CP problem, Phys. Lett. 138B, 150 (1984).

[2] P. Fayet, Supergauge invariant extension of the Higgs mechanism and a model for the electron and its neutrino, Nucl. Phys. B90, 104 (1975); 64B, 159 (1976); Phys. Lett. 69B, 489 (1977); 84B, 416 (1979); H. P. Nilles, M. Srednicki, and D. Wyler, Weak interaction breakdown induced by supergravity, Phys. Lett. 120B, 346 (1983); J. M. Frere, D. R. T. Jones, and S. Raby, Fermion masses and induction of the weak scale by supergravity, Nucl. Phys. B222, 11 (1983); J. P. Derendinger and C. A. Savoy, Quantum effects and $\mathrm{SU}(2) \times \mathrm{U}(1)$ breaking in supergravity gauge theories, Nucl. Phys. B237, 307 (1984); J. R. Ellis, J. F. Gunion, H. E. Haber, L. Roszkowski, and F. Zwirner, Higgs bosons in a nonminimal supersymmetric model, Phys. Rev. D 39, 844 (1989); M. Drees, Supersymmetric models with extended Higgs sector, Int. J. Mod. Phys. A 04, 3635 (1989).

[3] U. Ellwanger, C. Hugonie, and A. M. Teixeira, The next-tominimal supersymmetric standard model, Phys. Rep. 496, 1 (2010).

[4] T. Kobayashi, H. Makino, K. i. Okumura, T. Shimomura, and T. Takahashi, TeV-scale mirage mediation in NMSSM, J. High Energy Phys. 01 (2013) 081; K. Hagimoto, T. Kobayashi, H. Makino, K. i. Okumura, and T. Shimomura, Phenomenology of NMSSM in TeV-scale mirage mediation, arXiv:1509.05327.

[5] K. Choi, K. S. Jeong, T. Kobayashi, and K. i. Okumura, Little SUSY hierarchy in mixed modulus-anomaly mediation, Phys. Lett. B 633, 355 (2006); Phys. Rev. D 75, 095012 (2007).

[6] K. Choi, A. Falkowski, H. P. Nilles, M. Olechowski, and S. Pokorski, Stability of flux compactifications and the pattern of supersymmetry breaking, J. High Energy Phys. 11 (2004) 076; K. Choi, A. Falkowski, H. P. Nilles, and M. Olechowski, Soft supersymmetry breaking in KKLT flux compactification, Nucl. Phys. B718, 113 (2005).

[7] K. Choi, K. S. Jeong, and K. i. Okumura, Phenomenology of mixed modulus-anomaly mediation in fluxed string compactifications and brane models, J. High Energy Phys. 09 (2005) 039.

[8] M. Endo, M. Yamaguchi, and K. Yoshioka, A bottom-up approach to moduli dynamics in heavy gravitino scenario: superpotential, soft terms and sparticle mass spectrum, Phys. Rev. D 72, 015004 (2005).

[9] Y. B. Zeldovich, I. Y. Kobzarev, and L. B. Okun, Cosmological consequences of the spontaneous breakdown of discrete symmetry, Zh. Eksp. Teor. Fiz. 67, 3 (1974) [Sov. Phys. JETP 40, 1 (1974)].
[10] S. A. Abel, S. Sarkar, and P. L. White, On the cosmological domain wall problem for the minimally extended supersymmetric standard model, Nucl. Phys. B454, 663 (1995).

[11] K. Kadota, M. Kawasaki, and K. Saikawa, Gravitational waves from domain walls in the next-to-minimal supersymmetric standard model, J. Cosmol. Astropart. Phys. 10 (2015) 041.

[12] M. Kawasaki and F. Takahashi, Late-time entropy production due to the decay of domain walls, Phys. Lett. B 618, 1 (2005).

[13] M. Endo, K. Hamaguchi, and F. Takahashi, Moduli-Induced Gravitino Problem, Phys. Rev. Lett. 96, 211301 (2006); S. Nakamura and M. Yamaguchi, Gravitino production from heavy moduli decay and cosmological moduli problem revived, Phys. Lett. B 638, 389 (2006).

[14] Y. Kanehata, T. Kobayashi, Y. Konishi, O. Seto, and T. Shimomura, Constraints from unrealistic vacua in the nextto-minimal supersymmetric standard model, Prog. Theor. Phys. 126, 1051 (2011); T. Kobayashi, T. Shimomura, and T. Takahashi, Constraining the Higgs sector from false vacua in the next-to-minimal supersymmetric standard model, Phys. Rev. D 86, 015029 (2012).

[15] A. Vilenkin and E. P.S. Shellard, Cosmic Strings and other Topological Defects (Cambridge University Press, Cambridge, 2000).

[16] E. W. Kolb and M. S. Turner, The Early Universe (AddisonWesley, Reading, MA, 1990).

[17] W. H. Press, B. S. Ryden, and D. N. Spergel, Dynamical evolution of domain walls in an expanding universe, Astrophys. J. 347, 590 (1989).

[18] M. Hindmarsh, Level set method for the evolution of defect and brane networks, Phys. Rev. D 68, 043510 (2003).

[19] T. Garagounis and M. Hindmarsh, Scaling in numerical simulations of domain walls, Phys. Rev. D 68, 103506 (2003).

[20] A. M. M. Leite and C. J. A. P. Martins, Scaling properties of domain wall networks, Phys. Rev. D 84, 103523 (2011).

[21] A. M. M. Leite, C. J. A. P. Martins, and E. P. S. Shellard, Accurate calibration of the velocity-dependent one-scale model for domain walls, Phys. Lett. B 718, 740 (2013).

[22] T. Hiramatsu, M. Kawasaki, and K. Saikawa, On the estimation of gravitational wave spectrum from cosmic domain walls, J. Cosmol. Astropart. Phys. 02 (2014) 031.

[23] C. Panagiotakopoulos and K. Tamvakis, Stabilized NMSSM without domain walls, Phys. Lett. B 446, 224 (1999). 
[24] K. Hamaguchi, K. Nakayama, and N. Yokozaki, NMSSM in gauge-mediated SUSY breaking without domain wall problem, Phys. Lett. B 708, 100 (2012).

[25] M. Kawasaki, K. Kohri, and N. Sugiyama, MeV scale reheating temperature and thermalization of neutrino background, Phys. Rev. D 62, 023506 (2000).

[26] S. Hannestad, What is the lowest possible reheating temperature?, Phys. Rev. D 70, 043506 (2004).

[27] K. Ichikawa, M. Kawasaki, and F. Takahashi, The Oscillation effects on thermalization of the neutrinos in the Universe with low reheating temperature, Phys. Rev. D 72, 043522 (2005).

[28] D. G. Cerdeno, C. Munoz, and O. Seto, Right-hand sneutrino as thermal dark matter, Phys. Rev. D 79, 023510 (2009);
D. G. Cerdeno and O. Seto, Right-hand sneutrino dark matter in the NMSSM, J. Cosmol. Astropart. Phys. 08 (2009) 032.

[29] P. Sikivie, Axion cosmology, Lect. Notes Phys. 741, 19 (2008).

[30] P. Svrcek and E. Witten, Axions in string theory, J. High Energy Phys. 06 (2006) 051.

[31] H. Abe, T. Kobayashi, and H. Otsuka, Towards natural inflation from weakly coupled heterotic string theory, Prog. Theor. Exp. Phys. 2015, 63E02; Natural inflation with and without modulations in type IIB string theory, J. High Energy Phys. 04 (2015) 160.

[32] T. Asaka and M. Kawasaki, Cosmological moduli problem and thermal inflation models, Phys. Rev. D 60, 123509 (1999). 